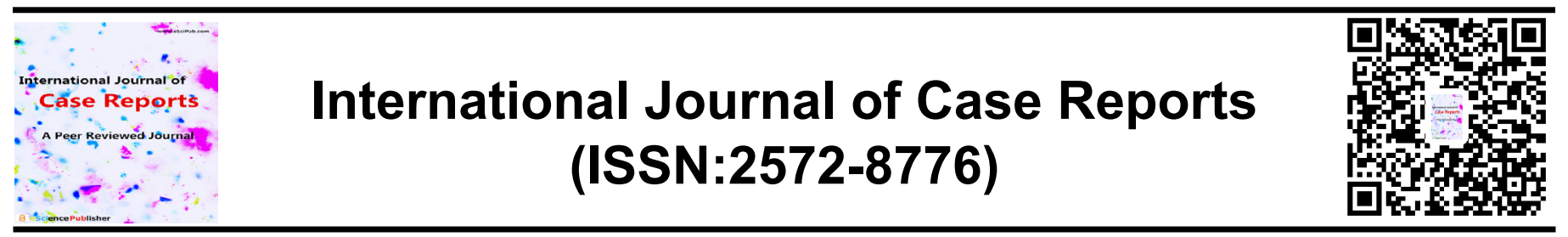

\title{
Cardiogenic Shock Due to Acute Myocarditis Following AZD1222 Vaccine Administration: a Case Report
}

\section{Rebeca Muñoz Rodríguez* , Aída Tindaya Igareta Herraiz, Martín Jesús García González}

Acute Cardiovascular Care Unit, Cardiology Department. University Hospital of the Canary Islands. San Cristóbal de La Laguna, Tenerife. Spain.

\section{ABSTRACT}

Aim: A number of cases of SARS-CoV-2-related myocarditis have been reported since the outbreak of the Covid-19 pandemia, with autoimmune-mediated damage due to molecular mimicry proposed as the virus' direct mechanism of injury. After commencing with a worldwide mass vaccination program, there have also been reports of myocarditis in the context of Covid-19 immunization. However, myocarditis is still not recognized as a potential side effect of the AZD1222 (ChAdOx1-S recombinant) vaccine administration by the health authorities.

Methods: In this article we report a case of myocarditis and further complications after vaccination for Covid-19 using the AZD1222 vaccine.

Conclusion: Even though it is hard to demonstrate the etiology of acute myocarditis, considering the cases reported around the world, the temporal association, the absence of other plausible causes, and the serological pattern described, it seems reasonable to assume that there is a genuine possibility of myocarditis being a side effect of AZD1222 vaccine administration.

Keywords: myocarditis, Covid-19, SARS-CoV-2, vaccination, immunization, thrombocytopenia, vaccine, AZD1222, mRNA vaccine
${ }^{*}$ Correspondence to Author:

Rebeca Muñoz Rodríguez

Acute Cardiovascular Care Unit, Cardiology Department. University Hospital of the Canary Islands. San Cristóbal de La Laguna, Tenerife. Spain.

How to cite this article:

Rebeca Muñoz Rodríguez* , Aída Tindaya Igareta Herraiz, Martín Jesús García González. Cardiogenic Shock Due to Acute Myocarditis Following AZD1222 Vaccine Administration: a Case Report. International Journal of Case Reports, 2021, 5:235,

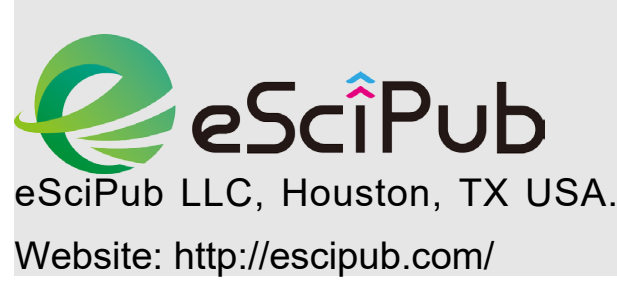




\section{INTRODUCTION}

Myocarditis is an underdiagnosed cardiovascular disease with a wide clinical spectrum ranging from asymptomatic cases to cardiogenic shock. Myocarditis may be caused by a great variety of infectious agents, particularly viruses. However, the etiology is unknown in most cases. Several cases of myocarditis had been associated with Covid-19 infection. ${ }^{[1]}$ Virus' direct injury, molecular mimicry between the spike-protein and selfantigens, as well as cytokine expression dysregulation have proposed as possible physiopathological mechanism. ${ }^{[8]}$

After initiating mass vaccination against Covid19 , some cases of myocarditis have been reported following the administration of the mRNA-1273 and BNT162b2 vaccines [2,3,4,8] without any evidence of concomitant Covid-19 infection. Moreover, recently, CDC Advisory Committee on Immunization Practices (ACIP) reported a "likely association" between the two Covid-19 mRNA vaccines mentioned before and cases of myocarditis and pericarditis ${ }^{[8]}$

The Ministry of Health of Israel, where one of the most advanced mass vaccination programs is underway, has reported 121 cases of myocarditis after 5,049,424 people received the second dose. Furthermore, the U.S. Centers for Disease Control (CDC), myocarditis and pericarditis rates are approximately 12.6 cases per million after receiving the second dose of mRNA vaccine among 12-39 year-olds, being more prevalent in males. ${ }^{[8]}$

At the time of writing, no cases of myocarditis related to AZD1222 have been reported. In this article we describe a case of myocarditis associated with the AZD1222 vaccine, as well as further, potentially related complications.

\section{MATERIALS AND METHODS}

A 68-year-old male, with a medical history of dyslipidemia and psoriasis and who received the first dose of the AZD1222 SARS-CoV-2 vaccine 37 days earlier, was referred to our emergency department with chest pain. He complained of an oppressive, intense pain in the chest with profuse sweating of two hours' presentation. We observed clinical signs of hypoperfusion and dynamic electrocardiogram changes with inferior and posterior ST-elevation. As an acute myocardial infarction was suspected, emergency angiography was performed without revealing evidence of coronary artery disease.

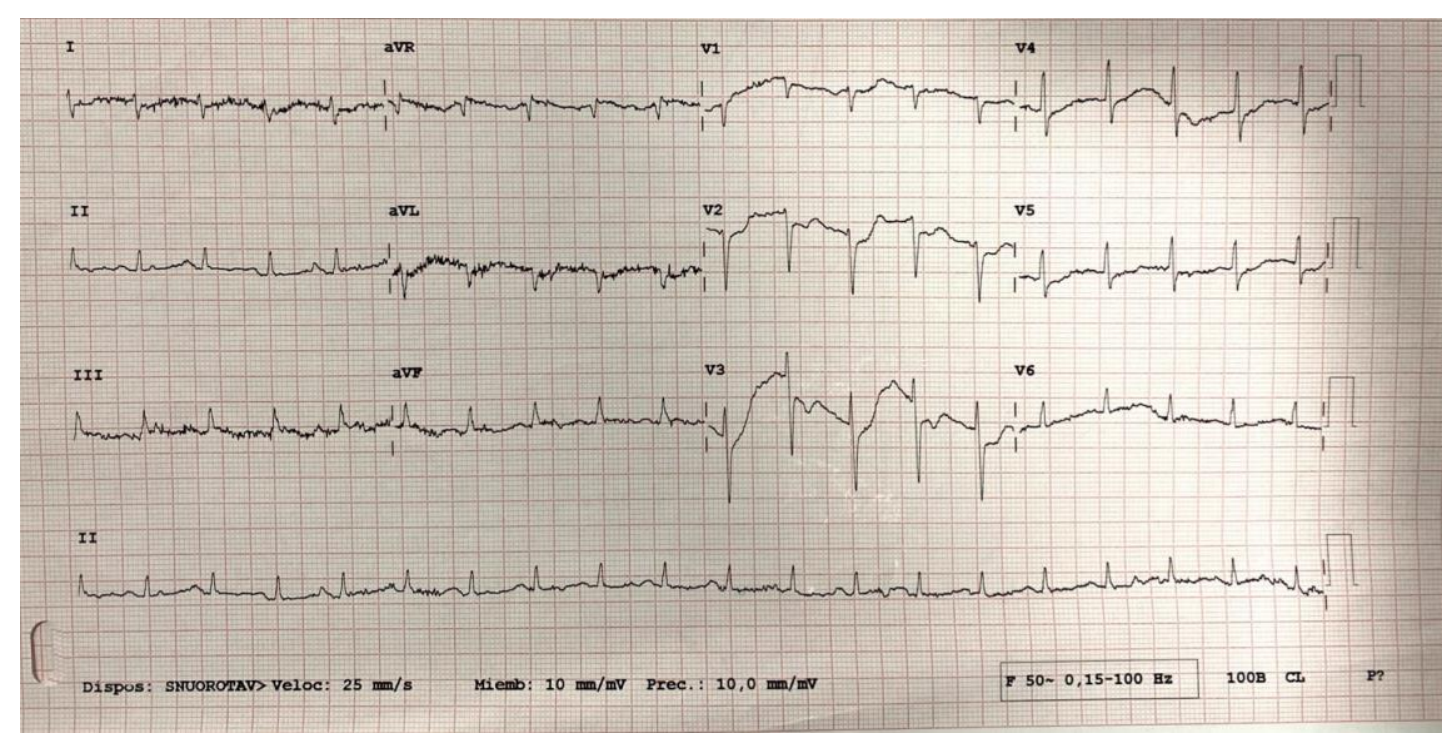

After the angiography, the patient was admitted required vasopressor and inotropic support. A to the Acute Cardiovascular Care Unit in a point-of-care ultrasound evidenced severe left situation of SCAl stage $\mathrm{C}$ cardiogenic shock and ventricular dysfunction with patchy regions of 
severe hypokinesis, moderate pulmonary hypertension, and moderate mitral regurgitation with a restrictive diastolic filling pattern. The laboratory results revealed elevated inflamematory markers with a C-reactive protein level of $88.83 \mathrm{mg} / \mathrm{L}$, neutrophilic leukocytosis, and elevated high-sensitivity troponin $\mathrm{T}$ of 1,982 $\mathrm{pg} / \mathrm{mL}$. The patient was Covid-19 PCR-negative. Serological testing demonstrated a typical
Covid-19 immunization pattern: IgG (spike) antibodies were positive (366.65 BAU/mL) and negative for IgG nucleocapsid. All tests performed to rule out immunological and infectious diseases were negative.

As the main suspicion was acute myocarditis, an MRI was performed and confirmed the diagnosis.

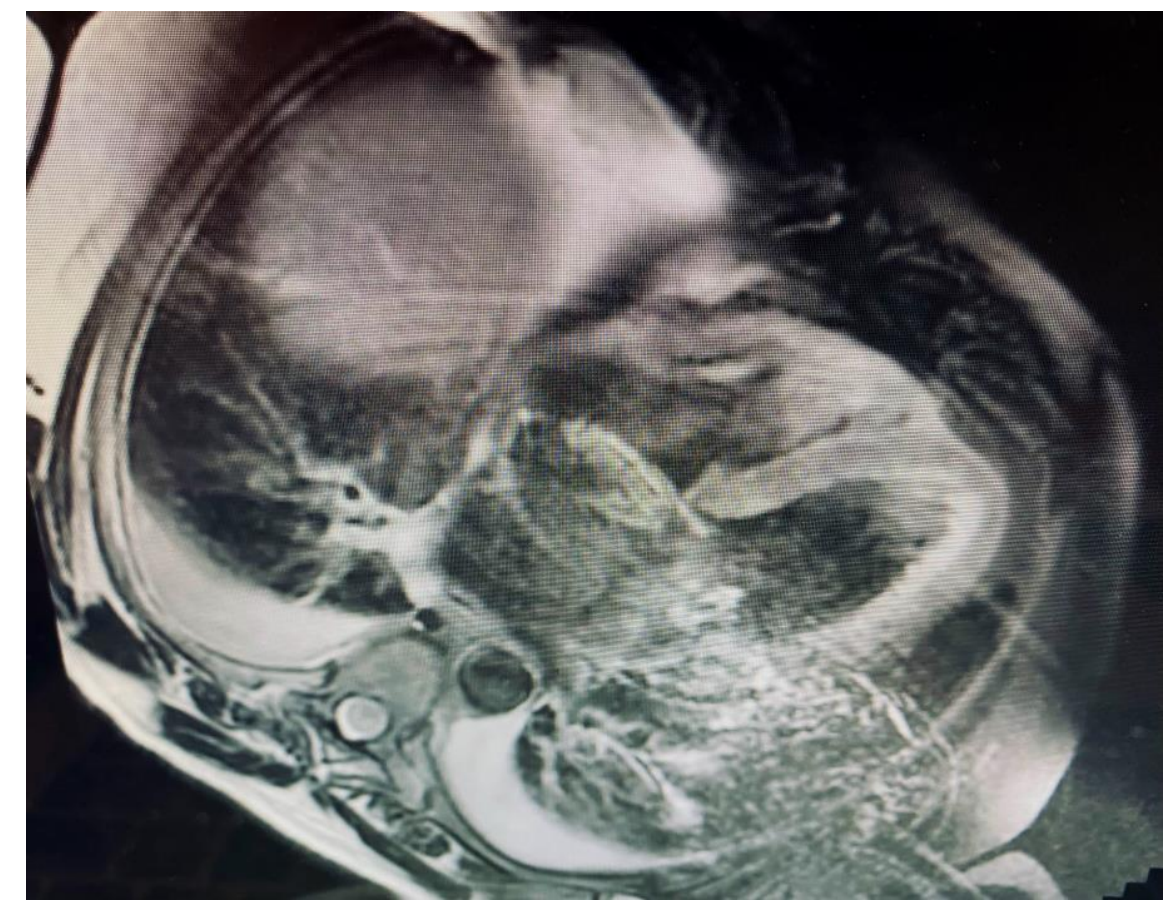

\section{RESULTS AND DISCUSSION}

The hemodynamic situation improved over the next few days, so the vasopressor agents were discontinued.

With respect to inflammation, the patient presented atrial fibrillation requiring the administration of unfractionated heparin. A formal ultrasound performed six days after admission showed a normalization of the ejection fraction, the continued presence of moderate pulmonary hypertension, and constant hypoxemia even in the absence of signs of heart failure. A pulmonary embolism was suspected and confirmed through CT angiography. In the meantime, the patient suffered a drastic decline in platelets $(33,000 \mathrm{x}$ $10^{3} / \mathrm{mm}^{3}$ ) and other acute thrombotic phenomena (right axillary artery thrombosis, superior vena cava thrombosis, and a right atrial thrombus), which was highly suggestive of heparin-induced thrombocytopenia. This suspicion was confirmed by the presence of anti-PF4 antibodies, so anticoagulation therapy was initiated with bivalirudin.

The patient had a satisfactory clinical evolution, platelet levels recovered, and no more thrombotic phenomena occurred. He was discharged after one month.

Although at this time 2.09 billion people have received at least one dose of a Covid-19 vaccine, the spectrum and prevalence of side effects is still under review. Several cases of myocarditis have been reported in the period shortly after a Covid-19 vaccination but were deemed to have a different underlying cause. $[6,7]$ In our case, all the tests performed in search of 
an infectious or immune-mediated cause proved negative, and serological testing demonstrated a typical pattern of SARS-CoV-2 immunization.

While the presence of PF4 antibodies, severe thrombocytopenia, and multiple thrombotic events can be justified by the administration of unfractionated heparin, vaccination with AZD1222 cannot be excluded as a precipitating factor (i.e., vaccine-induced thrombotic thrombocytopenia).

It is actually quite hard to demonstrate the etiology of this clinical spectrum. Yet considering the cases reported around the world, the temporal association, the absence of other plausible causes, and the serological pattern, it seems reasonable to assume that there is a significant possibility that the myocarditis in the present case is a side effect of AZD1222 vaccine administration.

Recently, myocarditis and pericarditis have been recognized to be a potential side effect of the mRNA-1273 and BNT162b2 vaccines by the Health Authorities. However, myocarditis is still not considered to be a side effect of the AZD1222 vaccine. More data and further study of this issue are necessary, as this complication may be an underdiagnosed entity requiring a higher level of suspicion. Physicians should be aware of this rare, potential side effect of AZD1222 vaccination in their clinical practice.

\section{CONFLICTS OF INTEREST:}

None declared.

\section{AUTHOR CONTRIBUTIONS:}

Aida Tindaya Igareta Herraiz and Rebeca Muñoz Rodríguez wrote and contributed equally to the article. Martín Jesús García González was the main attending physician and Head of the Acute Cardiovascular Care Unit at the University Hospital of the Canary Islands.

\section{REFERENCES}

[1]. Sawalha K, Abozenah M, Kadado A et al. Systematic Review of COVID-19 Related Myocarditis: Insights on Management and Outcome. Cardiovascular Revascularization
Medicine. 2021;23:107-113. doi:10.1016/j.c arrev.2020.08.028

[2]. Albert E, Aurigemma G, Saucedo J, Gerson D. Myocarditis following COVID-19 vaccination. Radiol Case Rep. 2021;16(8):2142-2145. doi:10.1016/j.radcr.2021.05.033

[3]. Kim H, Jenista E, Wendell D et al. Patients With Acute Myocarditis Following mRNA COVID-19 Vaccination. JAMA Cardiol. 2021. doi:10.1001/jamacardio.2021.2828

[4]. Marshall M, Ferguson I, Lewis $P$ et al. Symptomatic Acute Myocarditis in Seven Adolescents Following Pfizer-BioNTech COVID19 Vaccination. Pediatrics. 2021:e2021052478. doi:10.1542/ peds.2021-052478

[5]. https://www.gov.il/en/departments/news/0106202 1-03. Published 2021. Accessed July 1, 2021.

[6]. Bautista García J, Peña Ortega P, Bonilla Fernández J, Cárdenes León A, Ramírez Burgos L, Caballero Dorta E. Acute myocarditis after administration of the BNT162b2 vaccine against COVID-19. Revista Española de Cardiología (English Edition). 2021. doi:10.1016/ j.rec.2021.04.005

[7]. Shay D, Shimabukuro T, DeStefano F. Myocarditis Occurring After Immunization With mRNA-Based COVID-19 Vaccines. JAMA Cardiol. 2021. doi:10.1001/jamacardio.2021.2821

[8]. Bozkurt B, Kamat I, Hotez P. Myocarditis with COVID-19 mRNA Vaccines. Circulation. 2021. doi:10.1161/circulationaha.121.056135 